\title{
Massive star abundances in the Galaxy and the Magellanic Clouds
}

\author{
Stephen J. Smartt \\ Institute of Astronomy, University of Cambridge, \\ Madingley Road, Cambridge CB 3 OHA, UK
}

\begin{abstract}
The use of photospheric abundances in OB-type main-sequence stars and $\mathrm{A}$ and $\mathrm{B}$-type supergiants as probes of rotation and evolutionary status is reviewed. The abundances of $\mathrm{CNO}$ and boron can be compared quantitatively with stellar evolutionary calculations. In particular the abundance ratios of $\mathrm{N} / \mathrm{O}$ and $\mathrm{N} / \mathrm{C}$ can be derived in blue supergiants to determine if they are consistent with the stars having gone through a red supergiant phase and dredge-up of core material to the surface. The results from several different studies are reviewed and compared. For blue supergiant stars in the mass-range $5-80 \mathrm{M}_{\odot}$ the situation appears consistent - there is no evidence for stars of any mass having undergone blue-loops in the HR diagram. The stellar samples show significant signs of having $\mathrm{N}$ enriched atmospheres, and the quantitative values are consistent with stellar evolutionary calculations which invoke turbulent diffusive mixing while massive stars are on the main-sequence. Results on the interesting blue supergiant Sher 25 are presented, and linked to $\mathrm{Sk}-69^{\circ} 202$; the B3Ia progenitor of SN 1987A. A spectacular ejection nebula surrounds Sher 25, much like that which was ejected by Sk $-69^{\circ} 202$ during its final stages of evolution. Both of these were thought to have been formed during a mass-loss event when the stars were in the red supergiant phase. The CNO abundances derived in Sher 25 suggest the nebula was ejected during the blue supergiant phase, and that rotation can explain the $\mathrm{N}$-enrichment found in the stellar photosphere.
\end{abstract}

\section{The metal abundance gradients in the Milky Way}

The B-type main-sequence stars have been used extensively over the last twenty years to measure the present day abundances in Galactic young clusters and the field. The early B-type stars have main-sequence masses of typically 10 $15 \mathrm{M}_{\odot}$, and absolute magnitudes of up to -4 mag. They can be observed at high spectral resolution, with $4 \mathrm{~m}$ telescopes out to approximately $18 \mathrm{kpc}$ from the Galactic centre (Smartt, Dufton \& Rolleston 1996). Both LTE and nonLTE methods have been used to determine stellar atmospheric parameters and photospheric abundances. While non-LTE methods do provide more accurate absolute abundances, Rolleston et al. (2000) and Smartt \& Rolleston (1997) have shown that a consistent, homogeneous analysis in LTE are certainly capable of accurately measuring abundance gradients in the Milky Way. The key point is that the analysis must be done in a consistent manner on an extensive data set, rather than incorporating results from many different studies and authors. These two papers derive an oxygen, silicon and magnesium abundance gradients 
of $-0.07 \pm 0.01 \mathrm{dex}_{\mathrm{kpc}}^{-1}$, which is now in excellent agreement with the $\mathrm{H}$ II and planetary nebulae oxygen results (see extensive comparisons in Rolleston et al. 2000). This value of -0.07 appears to be the canonical value for the Galactic abundance gradient for oxygen (but see Daflon \& Cunha, these Proceedings). One assumes that the $\mathrm{O}, \mathrm{Si}$ and $\mathrm{Mg}$ photospheric abundances have not been altered in any way during main-sequence evolution. While this is undoubtedly true for $\mathrm{Si}$ and $\mathrm{Mg}$, any mixing of core gas to the surface could adjust the $\mathrm{O}$ abundance. However stellar evolutionary calculations suggest that for stars in this mass-range, any reasonable amounts of mixing would affect $\mathrm{O}$ by around 0.1 dex, which is below the systematic and statistical uncertainties in the derived abundances.

\section{CNO and boron abundances in main-sequence B-type stars}

For several years now the evolution of massive stars has been thought to be a function of not only mass and metallicity, but also rotation. Evolutionary calculations by many authors (e.g., papers by Maeder and Meynet, these Proceedings) have suggested that rapid rotation can induce mixing of core gas into the stellar atmosphere by turbulent diffusion. As this gas has been through the CN and NO cycles, it is likely to be rich in nitrogen and depleted in carbon and oxygen. As pointed out in Section 1, the oxygen depletion is likely to be below our current uncertainties in abundance determinations.

Hence determining the CNO abundances in a sample of stars with the same initial composition (e.g., stars in a young OB-association) would allow the dispersion of the $\mathrm{N}$ abundances (in particular) to be probed in order to determine if there is any correlation with rotational velocity or age. Obviously one can only measure the projected component of the intrinsic rotational velocity, and hence it is necessary to observe and analyse a large number of stars to determine any trend (and assume the rotational axes are randomly distributed). The large sample of stars $(\sim 80)$ in Rolleston et al. (2000) is not particularly useful, as the gradient effect complicates any possible trend. The most extensive analyses of young stars in a cluster has been presented in Daflon et al. (2001) who presented the $\mathrm{N} / \mathrm{O}$ abundances of 17 young B-type main-sequence stars in Cep OB2. Although the two stars with highest $\mathrm{N} / \mathrm{O}$ are amongst the group of stars with highest projected rotational velocities, there is no convincing evidence of a trend in N/O with $v \sin i$. The studies of Cunha \& Lambert (1994) and Gies \& Lambert (1992) also showed no compelling evidence of a link between either $v \sin i$ or age with nitrogen enhancement. One really requires a larger sample of stars in young stellar clusters of the same initial metallicity to significantly improve the situation. With the upcoming flames spectrograph on the VLT, it will be possible to gather spectra of 130 stars simultaneously at high-resolution, which should see a huge observational advance in this area.

Recent work on determining the boron abundances in Galactic B-type stars has suggested that stars which are N-rich and B-poor do exist (Venn et al. 2002), with excellent quantitative agreement with evolutionary models incorporating mixing. This experimental test is a strong indicator that mixing does occur in a fraction of stars. However there do exist stars with normal $\mathrm{B}$ and $\mathrm{N}$ abundances, which suggests that these are not pole-on fast rotators but have intrinsically low rotational velocities and little turbulent mixing. Unfortunately B is only visible 
Table 1. The N/O or N/C values measured (by number) in blue supergiants in the range $5-60 \mathrm{M}_{\odot}$, compared with the theoretical values. The A-type measured and theoretical values are from Venn (1995); all other theo retical values and LBV measured ratios from Lamers et al. (2001); B-type supergiants and Sher 25 from McErlean et al. (1999) and Smartt et al. (2002); SN 1987A from Fransson et al. (1989). For reference, the solar values are $\mathrm{N} / \mathrm{O}=0.14$ and $\mathrm{N} / \mathrm{C}=0.41$ (Grevesse \& Sauval 1998).

\begin{tabular}{lcc|cc}
\hline \hline & $\begin{array}{c}\text { mass } \\
\left(\mathrm{M}_{\odot}\right)\end{array}$ & ratio & measured & theoretical \\
\hline A-type supergiants & $5-20$ & $\mathrm{~N} / \mathrm{C}$ & 2.4 & $4-10$ \\
B-type supergiants & $20-50$ & $\mathrm{~N} / \mathrm{O}$ & $0.1-0.5$ & $1-20$ \\
LBVs & $60-80$ & $\mathrm{~N} / \mathrm{O}$ & $0.1-6$ & $10-50$ \\
Sher 25 & 60 & $\mathrm{~N} / \mathrm{O}$ & 0.4 & $3-20$ \\
SN 1987A & 20 & $\mathrm{~N} / \mathrm{O}$ & 1.6 & $\sim 1$ \\
\hline \hline
\end{tabular}

in the UV (with HST-STIS), ruling out the possibility of a survey of large numbers of stars.

\section{Using abundances to probe the evolutionary status of evolved massive stars}

Stars of initial mass greater than $\sim 15 \mathrm{M}_{\odot}$ will be O-types on the main-sequence and deriving $\mathrm{CNO}$ abundances in early and mid O-types is extremely difficult. They are often very fast rotators, the metal lines are weak and often in emission, making unified models which treat the wind and photosphere together in a spherical non-LTE framework with line-blanketing essential. Only the O9 stars have so far yielded reasonable CNO abundances (Villamariz et al. 2002). This was a study restricted to four Galactic $\mathrm{O} 9$ stars, three of which show fairly normal He and CNO abundances (similar to B-type stars), and one of which shows some indication of $\mathrm{N}$-enrichment from rotationally induced mixing. Hence to date, the amount of mixing in main-sequence objects of masses higher than $\sim 15-20 \mathrm{M}_{\odot}$ is not well constrained by surface abundance studies.

When these massive stars evolve they move into the blue supergiant region to produce the A and B-type supergiants, with luminosity dependent on their initial mass. These stars lend themselves better to optical abundance studies due to their cooler temperatures, brighter visual magnitudes and narrower line widths. However theoretical models have trouble in predicting the characteristics of this population. For example the B/R supergiant ratio and its variation with metallicity has not been consistently reproduced (Langer \& Maeder 1995). The Geneva tracks don't predict that large numbers of massive B-type supergiants should exist, but they do predict that A-type supergiants at solar metallicity exist as blue-loop objects (Meynet et al. 1994). If the A or B-type stars have been in a previous red supergiant phase the structure models predict that they must undergo dredge up, and hence their photospheres will become significantly contaminated with the products of core CNO cycle H-burning. However the A-type supergiants studied by Venn (1995) have ratios $\mathrm{N} / \mathrm{C} \simeq 2$, whereas the models 
predict $\mathrm{N} / \mathrm{C}$ in the range $4-10$ for post-RSG objects. The values are consistent with some form of mixing on or just after the main-sequence phase, and suggest that the stars have not been in the red supergiant phase and undergone blue-loops. The initial mass of these stars was $5-20 \mathrm{M}_{\odot}$.

McErlean et al. (1999) presented spectra of 46 Milky Way B-type supergiants, and looked at the global trend of the CNO line strengths. There appears to be a large range in the $\mathrm{N} / \mathrm{C}$ line strength ratio (note that $\mathrm{N} / \mathrm{C}$ abundances were not derived in each individual star), with approximately half the stars showing $\mathrm{N} / \mathrm{C}$ line strengths indicative of some form of processed gas appearing in the atmosphere. In a recent analysis of the blue supergiant Sher 25 (see Section 4), Smartt et al. (2002) also quantitatively analysed three of the McErlean et al. B-type supergiants, finding that $\mathrm{N} / \mathrm{O}$ was somewhat higher than solar. Calculations of the expected values of $\mathrm{N} / \mathrm{O}$ in blue supergiants which have been through the red supergiant phase (and convective dredge-up) have been recently presented by Lamers et al. (2001). The measured N/O in the B-type supergiants are significantly lower than those expected for post-RSG stars. The range of measured values, and those calculated by Lamers et al. (2001) are presented in Table 1. Two LMC and two Galactic LBV nebulae were also analysed in the latter paper, and their $\mathrm{N}$ abundances compared to $\mathrm{O}$ and $\mathrm{S}$. Lamers et al. found that the measured values are again significantly lower than those predicted if the stars had gone through dredge-up in the red supergiant region (see Table 1). This suggests that they have not previously been red supergiants and that the nebulae are ejected during the blue supergiant phase. The chemical enhancements seen in these three cases are possibly all due to some form of rotation induced mixing, which will have some broad distribution based on initial rotational velocities and mass-loss rates. The results from the A-type supergiants and B-type supergiants are also consistent with a range of rotationally induced mixing occurring on the main-sequence.

\section{CNO abundances in the $60 \mathrm{M}_{\odot}$ supergiant Sher 25}

Sher 25 is a B1.5Ia supergiant in the massive Galactic cluster NGC 3603. It is surrounded by a circumstellar nebula, discovered by Brandner et al. (1997a) and beautifully illustrated in $H S T$ press release images STScI-PRC99-20 ${ }^{1}$. It consists of a ring-shaped equatorial emission line nebula (expansion velocity of $20 \mathrm{~km} \mathrm{~s}^{-1}$ ) and probable bipolar outflows to the northeast and to the southwest (expansion velocity of $83 \mathrm{~km} \mathrm{~s}^{-1}$ ). The ring and the bi-polar ejecta have roughly the same dynamical age which would suggest a common origin in a stellar outburst $\sim 6600$ yrs ago. Sher 25 is currently a hot blue supergiant and Brandner et al. have suggested that the star lived briefly for a period as a red supergiant during which time it ejected the nebula. Given the fact that the studies of Galactic massive stars listed in Table 1 suggest that stars in the range $5-80 \mathrm{M}_{\odot}$ have not previously been red supergiants, it is of great interest to determine the CNO abundances of Sher 25.

In addition it has been suggested (by Brandner et al. 1997b) that Sher 25 could be a twin of the progenitor of SN 1987A, which was a B3Ia supergiant

\footnotetext{
${ }^{1}$ http://oposite.stsci.edu/pubinfo/PR/1999/20/index.html
} 


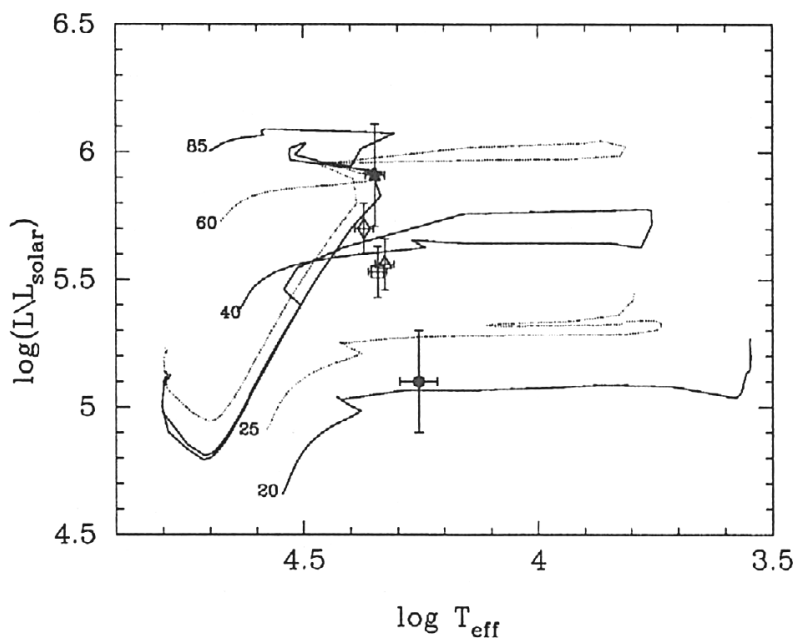

Figure 1. Stellar evolutionary tracks for stars with zero-age main sequence masses of 20, 25, 40, 60 and $85 \mathrm{M}_{\odot}$ from Meynet et al. (1994). The symbols are: Sher 25 (filled star), Sk-69 202 progenitor of SN 1987A (filled circle). The stars HD 2905 (open diamond), HD 13854 (open square), HD 14956 (open triangle) were used in the comparison analysis of Smartt et al. (2002).

(Walborn et al. 1989). This is due to the existence of a similar ring structure now seen around SN 1987A which in existence before the SN explosion and hence was ejected by the progenitor star. Fransson et al. (1989) suggested that it had passed through a previous RSG phase given the CNO line strengths in the earlytime UV spectra, although the uncertainties are large.

Smartt et al. (2002) have presented high-resolution optical spectra of Sher 25 taken with the Anglo-Australian Telescope and the UCLES spectrometer. A model atmosphere analysis and wind analysis gave the atmospheric parameters: $T_{\text {eff }}=22300 \mathrm{~K}, R=59 \mathrm{R}_{\odot}, \log g=2.6, \dot{M}=1.75 \times 10^{-6} \mathrm{M}_{\odot} \mathrm{yr}^{-1}$, and log $L / \mathrm{L}_{\odot}=5.9$. Comparing it with the evolutionary tracks of Meynet et al. (1994) results in a mass determination of approximately $60 \mathrm{M}_{\odot}$ (see Figure 1 ).

Three early B-type stars of very similar atmospheric parameters were chosen from the McErlean et al. sample, and were analysed in an identical manner to Sher 25. The $\mathrm{Mg}$ and $\mathrm{Si}$ abundances in Sher 25 are very close to stars in the solar neighbourhood, which is to be expected given that the distance from the Galactic centre $\left(R_{\mathrm{g}} \simeq 8.5 \mathrm{kpc}\right)$ is very similar to that of the Sun. Hence the CNO abundances of Sher 25 can be directly compared with other Galactic B-type supergiants to determine if there is anything unusual in its chemical composition. The N/O ratio is given in Table 1 and is very similar to that of the group of stars in the McErlean et al. study which show the largest sign of CNO processed gas in their atmospheres. However it is a factor of at least seven below what we would expect if the star had been through a previous red supergiant phase. Hence we are forced to conclude that this star has never been a red supergiant, and has not undergone first dredge-up. It appears that the nebula was ejected during the blue period of its life - which is similar to the conclusions for the ejection time of LBV nebulae in Lamers et al. (2001). 
The value derived for $\mathrm{N} / \mathrm{O}$ is higher than solar and higher than that in unevolved B-type stars in the solar neighbourhood. Comparing it with the calculations of Lamers et al. (2001) it appears consistent with rotationally induced mixing having occurred when the star was on, or near, the main-sequence. For a $60 \mathrm{M}_{\odot}$ star the $\mathrm{N} / \mathrm{O}$ ratio in the atmosphere of a blue supergiant which has undergone rotationally induced mixing is in the range of $1<\mathrm{N} / \mathrm{O}<3$ if $\tau_{\text {mix }}$ (the mixing timescale introduced by Lamers et al.) is $2-4$ times the main sequence lifetime. The value for Sher $25(\mathrm{~N} / \mathrm{O}=0.4)$ is lower than predicted values for $2<\tau_{\operatorname{mix}}<4$, but is a factor of three higher than the expected initial value of $\mathrm{N} / \mathrm{O}=0.12$. This suggests that Sher 25 has undergone less mixing than the Lamers et al. models predict, although almost certainly some moderate amount of mixing has occurred to enhance the $\mathrm{N}$ abundance. It could be interpreted that a larger mixing time (i.e., greater than 2-4) is more appropriate for Sher 25.

Sher 25 shows many similarities to Sk-69\%202, the progenitor of SN $1987 \mathrm{~A}$. The inner ring in the circumstellar nebula around Sher 25 is similar in mass, age and kinematics to that seen around SN 1987A. The latter was in existence before the supernova exploded, and is a feature of the late stages of evolution of $\mathrm{Sk}-69^{\circ} 202$ and was flash ionized by the supernova explosion. The chemical composition of the ring system around SN 1987A was measured by Fransson et al. (1989) from IUE spectroscopy - which targeted the narrow emission lines of the circumstellar gas. The $\mathrm{N} / \mathrm{O}$ ratio is listed in Table 1 . It is interesting to note that it is considerably higher than any $\mathrm{A}$ or B-type supergiant we know of in the Galaxy or LMC. If the CNO abundances measured by Fransson et al. are reliable, and representative of the photospheric abundances in the late stages of the life of Sk-69 202 , then we do not know of a single blue supergiant counterpart to this star. Sher 25 has much lower N/O values in its atmosphere, although the gaseous abundances in the ionized nebula should be measured. Why do we not see any B-type stars with $\mathrm{N} / \mathrm{O}$ values high enough to be similar to that measured in the nebular around SN 1987A? The fact that Sk-69 202 stands alone in its $\mathrm{N} / \mathrm{O}$ raises some doubt over the abundances derived in the early IUE spectra, and it would be highly desirable to check this model.

\section{Conclusion - no evidence for blue loops at solar metallicity}

The CNO abundances in evolved massive stars of masses in the range $5-80 \mathrm{M}_{\odot}$ have been summarised. These stars are A and B-type supergiants and LBVs. In all cases it is found that there is clear evidence for nitrogen enhancement in the atmospheres of many stars in the samples. However the nitrogen enhancement (measured by either $\mathrm{N} / \mathrm{C}$ or $\mathrm{N} / \mathrm{O}$ ) is not high enough to suggest that any of these stars have been through a red supergiant stage and undergone dredge-up. The results are compatible with some degree of rotationally induced mixing having occurred while the stars were on or near the main-sequence. The quantitative evolutionary calculations of Lamers et al. (2001) which incorporate diffusive mixing on the main-sequence, and reasonable values for stellar mass loss, agree well with the observationally determined values. The fact that we see no evidence for blue-loops occurring in any mass range at solar metallicity should be some cause for concern for stellar evolutionary models. Particularly as most models (e.g., the Geneva tracks) do still predict $5-15 \mathrm{M}_{\odot}$ A-type supergiants should be 
blue-loop objects, and the timescale for the existance of the more massive B-type supergiants is extremely short.

Abundance determinations of $\mathrm{CNO}$ and boron in late $\mathrm{O}$ and $\mathrm{B}$-type mainsequence stars also suggest that rotationally induced mixing occurs on the mainsequence in some stars. But the distribution of this mixing, the direct relationship with rotational velocity and its magnitude are still not observationally well constrained.

The observational situation should improve dramatically with the commissioning of the FLAMES spectrometer ${ }^{2}$ on the VLT. This will allow simultaneous multi-object spectroscopy of 130 stars, at resolutions up to $17 \mathrm{~km} \mathrm{~s}^{-1}(R=17000)$. It will hence produce large numbers of stellar spectra of high-resolution and high signal-to-noise in young Galactic clusters, the LMC and SMC. We will be able to look for trends in CNO abundance with metallicity, age and rotational velocity on a statistically sure footing for the first time. It will allow A and B-type supergiant populations to be studied in detail in the same fields and clusters, provding excellent constraints for theoretical models.

\section{References}

Brandner, W., Grebel, E.K., Chu, Y.H., Weis, K. 1997a, ApJ (Letters) 475, L45

Brandner, W., Chu, Y.H., Eisenhauer, F., et al. 1997b, ApJ (Letters) 489, L153

Daflon, S., Cunha, K., Butler, K., Smith, V. 2001, ApJ 563, 325

Fransson, C., Cassatella, A., Gilmozzi, R., et al. 1989, ApJ 336, 429

Grevesse, N., Sauval, A.J. 1998, in: C. Frölich, M.C.E. Huber, S.K. Solanki \& R. von Steiger (eds.), Solar Composition and its Evolution - From Core to Corona, Space Sci. Reviews 85, 161

Lamcrs, H, Nota, A., Panagia, N., Smith, L.J., Langcr, N. 2001, ApJ 551, 764

Langer, N., Maeder A. 1995, A\&A 295, 685

McErlean, N.D., Lennon, D.J. \& Dufton, P.L. 1999, A\&A 349, 55

Meynet, G., Maeder, A., Schaller, G., et al. 1994, A\&AS 103, 97

Rolleston, W.R.J., Smartt, S.J., Dufton, P.L., Ryans, R.S.I. 2000, A\&A 363, 537

Smartt, S.J., Dufton, P.L., Rolleston, W.R.J. 1996, A\&A 305, 164

Smartt, S.J., Rolleston, W.R.J. 1997, ApJ (Letters) 481, L47

Smartt, S.J., Lennon, D.J., Kudritzki, R.-P., et al. 2002, A\&A 391, 979

Venn, K.A. 1995, ApJ 449, 839

Villamariz, M.R., Herrero, A., Becker, S.R., Butler, K. 2002, A\&A 388, 940

Walborn, N.R., Prevot, M.L., Prevot, L., et al. 1989 A\&A 219, 229

\section{Discussion}

MEYNET: Is there any evidence for Sher 25 to belong to a binary system?

SMARTT: Good question; we have spectra from one night only, separated by 1 - 2 hours. Over this timescale there is no evidence of radial velocity variations, but we need to monitor this over the days-months timeframe to check for any sign of binarity. It hasn't so far been done.

\footnotetext{
${ }^{2}$ http://ww. eso.org/instruments/flames
} 
SONNEBORN: The evidence that Sher 25 is an analogue for $\mathrm{Sk}-69^{\circ} 202$ (the progenitor of SN 1987A) is entirely circumstantial, based on the geometry of the elliptical circumstellar ring. The CNO abundances of the ring near Sher 25 must be demonstrated to be similar to the 1987A ring for the post-RSG analogy to be valid.

SMARTT: I agree it is important for the CNO abundances in the equatorial ring around Sher 25 to be derived. This hasn't yet been done quantitatively, the [N II] line strength has been measured but there are not enough lines available for abundance measurements.

LANGER: It is nice to see that your results support the idea of rotationally induced mixing in massive stars. However, current massive star models, in particular also those with rotation, predict blue loops, which contradicts your findings. To André Maeder: does this not bring our models in trouble?

SMARTT: To check this in details, I think we should observe abundances and rotational velocities for B-type supergiants in clusters. There the constraints from the HR diagram and abundances would be stronger. Now, due to the large scatter in rotational velocites, there is a large scatter in the extension of the MS tracks and some B-type supergiants might still be on the MS, sitting near the top of these extended tracks. I noticed that the tracks by Langer extend much farther than yours and would make this possibility even more likely.

WALBORN: It is quite possible that Sher 25 is in the foreground of NGC 3603, in which case it is less massive. The cluster is $7 \mathrm{kpc}$ away and is much younger than Sher 25.

SMARTT: Sher 25, if it is at the distance of NGC 3603, is $\sim 60 \mathrm{M}_{\odot}$, which is similar to the most massive O-stars. Given the uncertainties in the masses of these, their evolutionary lifetimes, and some reasonable extended formation timescale, I think Sher 25's position in the HR diagram is quite consistent with membership of NGC 3603.

EL EID: Concerning the A-type supergiants, the sodium enrichment should be discussed together with nitrogen enrichment. These stars seem to show enhanced sodium enrichment in their atmosphere, but this enhancement is still uncertain.

SMARTT: I don't know if the sodium abundances in A-type supergiants are reliably enough determined to allow such a quantitative comparison. I presume they are not.

MAEDER: The situation looks rather clear for B- and A-type supergiants. As to MS B-type stars, do I understand correctly that there are some evidences of N-enrichments for MS B-type stars in the SMC, as suggested by Danny Lennon?

SMARTT: There is some marginal evidence that this is true, but I think we need significantly more stars and CNO analyses of these stars to draw quantitative conclusions. This should happen with the VLT-FLAMES spectrograph.

HumphreYs: Why do you say Sher 25 (in NGC 3603) is a post-RSG? With an initial mass of $\sim 60 \mathrm{M}_{\odot}$ it has probably not been an RSG. And you also show that it's abundances are consistent with rotational mixing as a BSG. The observed ring is more likely from an unstable state (LBV?) on the blue side of HRD.

SMARTT: It has been classified or suggested to be, a post-RSG by previous authors simply on the basis of the existence of the circumstellar nebula. I would accept it could never have reached the RSG phase, and the nebula was ejected in some sort of unstable or LBV state. 